\title{
Ultrasound Monitoring and Age Soniographic Characteristics of Community-Acquired Pneumonia in Children
}

DOI: $10.17691 / \mathrm{stm} 2015.7 .2 .15$

Received December 1, 2014

T.I. Dianova, Assistant, Pediatrics Department, Medical Faculty';

D.V. Safonov, MD, DSc, Professor, Department of Radiodiagnosis, Postgraduate Faculty²

${ }^{1}$ Chuvash State University named after I.N. Ulianov, 45 Moskovshy Avenue, Cheboksary, 428017, Chuvash Republic,

Russian Federation;

${ }^{2}$ Nizhny Novgorod State Medical Academy, 10/1 Minin and Pozharsky Square, Nizhny Novgorod, 603005,

Russian Federation

The aim of the investigation was to study ultrasound semiotics of community-acquired pneumonias in children of different age, and its dynamics in the course of treatment.

Materials and Methods. Pneumonia semiotics study and dynamic monitoring of the disease have been carried out on the basis of chest ultrasound of 154 children divided into 4 age groups: 14 infants (9.1\%) from birth to 3 months; 60 children (39.0\%) from 3 months to 3 years of age; 49 children $(31.8 \%)$ from 4 to 7 years of age; 31 children $(20.1 \%)$ from 7 to 18 years of age.

During the hospital stay the children underwent ultrasound at least three times: on admission to determine pneumonia volume and localization; on day 3-5 or day 5-7 to assess the dynamics of sonographic picture and the necessity of etiotropic therapy change; before discharge to evaluate residual effects and determine the follow-up strategy.

Ultrasound revealed zones of airness impairment of various size in the pulmonary tissue of all children. Pneumonia semiotics presented extensive and lobar forms of pneumonia. An extensive type occupies a segment or a lobe, and looks like a delta-shaped air-free area with fuzzy contours and heterogeneous structure, with an air echobronchogram, and occurs in children of older age. Lobar pneumonia presents small rounded subpleural infiltrate up to $15 \mathrm{~mm}$ with a homogeneous hypoechogenic structure, and is typical of newborns and younger children.

The size and echostructure of apneumatic (air-free) area were the assessment criteria of pneumonia regression.

Conclusion. Chest ultrasound is an informative radiologically safe technique for diagnosing and dynamic control of pneumonia in children of different age, which enables timely assessment of etiotropic therapy efficacy.

Key words: pulmonary ultrasound diagnosis; pneumonia; pulmonary diseases in children; air echobronchogram.

Community-acquired pneumonia is an acute infectious pulmonary disease of different (mainly bacterial) etiology developing outside the hospital or in the first $48-72 \mathrm{~h}$ of hospital stay and accompanied by fever and symptoms of lower respiratory tract lesion (dyspnea, cough and crepitation) with the presence of infiltrative changes on the roentgenogram. Its incidence rate in infants of the first year of life is about $1-3 \%$, it reaching $35-40 \%$ in children aged 2 to 4 years [1, 2].

The key radiological method for pneumonia diagnosis in children as well as adults is a double-view chest X-ray. However, X-ray examination is not always informative: in $2-5 \%$ of patients the $\mathrm{X}$-ray picture is atypical, or the clinical signs similar to those of pneumonia are caused by some other pathological process [1]. In such cases computed tomography (CT) is required, though additional exposure to a harmful radiation is particularly critical for children. Chest ultrasound can be a good alternative to radiological methods for pneumonia diagnosis.

Ultrasound examination of the lungs and pleural cavities is available not only in hospitals but in outpatient departments as well, because it can be carried out on a real-time basis using any ultrasound equipment and without specific preparation of a child. In clinical practice transthoracic ultrasound is commonly used for detection of pleural effusion, but it is rarely used to diagnose pulmonary pathology because of a widespread opinion that its informative value is rather low. However, the pulmonary tissue containing air is not visualized only in case of complete reflection of ultrasound from the air in subpleural alveoli; when airness is lost, the lung parenchyma is well sounded revealing all pathological changes. Pneumonia semiotics in adults has been studied in detail [3, 4], however, investigations of it in children have just begun [5-7]: ultrasound characteristics considering the age pulmonary morphology have not been determined, the dynamics of ultrasound image of resolving inflammatory infiltrate has not been monitored, ultrasound examination algorithm has not been worked out.

The aim of the investigation was to study ultrasound semiotics of community-acquired pneumonias in children of different age and its dynamics in the course of treatment.

Materials and Methods. The study is based on the findings of chest ultrasound in 154 children

For contacts: Dianova Tatiyana Ivanovna, e-mail: d.t.i_21@mail.ru 
who underwent treatment for community-acquired pneumonia in Republican Children's Teaching Hospital of Cheboksary in 2013-2014, including 87 boys (56.5\%) and 67 girls (43.5\%) aged from birthday to 18 . The children were admitted to the hospital at different periods of the disease: 64 children (41.6\%) were hospitalized on day 1-5 from the onset of pneumonia, 38 children $(24.7 \%)$ on day $5-10,32$ children $(20.8 \%)$ on day $10-15$, 13 children $(8.4 \%)$ on day $15-20,7$ children $(4.5 \%)$ on day 20-25 of the disease, therefore observation started at different stages of the inflammatory process running with different severity. Before admission, 52 children $(33.8 \%)$ received etiotropic therapy, which appeared to be ineffective.

The study was carried out in accordance with Declaration of Helsinki (adopted in June 1964 (Helsinki, Finland) and revised in October 2000 (Edinburgh, Scotland)) and was performed following approval by Ethics Committee of Nizhny Novgorod State Medical Academy. Informed consent was received from the patients' parents.

For detailed analysis of pneumonia echosemiotics the children were divided into four groups according to the age-related pulmonary morphology.

Group 1 included 14 infants $(9.1 \%)$ from birth to 3 months, among them 9 boys and 5 girls. The lung structure in children of this age group preserves the features characteristic of fetal lungs: a great amount of loose connective tissue and a weak development of elastic frame, a more pronounced fibrous system of pleura and interlobular septa.

Group 2 consisted of 60 children (39.0\%) aged 3 months to 3 years, including 38 boys and 22 girls. This is a period of intensive growth of the lung lobes and bronchopulmonary segments with the increase of the length and width of respiratory bronchi and alveolar ducts and the volume of functioning alveoli; separate segments are equalizing in volume and maturity.

Group 3 consisted of 49 children (31.8\%) 4 to 7 years of age, including 25 boys and 24 girls. The process of differentiating the elements of bronchopulmonary system is coming to an end, the connective tissue in septa disappears almost completely, the elastic frame of the lungs is being accomplished, acini formation is being over. An important characteristic feature is formation of interalveolar connections, the Pores of Kohn, which play an essential role in collateral ventilation in bronchial patency impairment.

Group 4 consisted of 31 children (20.1\%) 7 to 18 years of age, including 15 boys and 16 girls. The lungs are formed and correspond to those of an adult in appearance and architectonics.

To perform chest ultrasound we used ultrasound scanners Hitachi Vision Avius (Japan) and SonoScape S8Exp (China) with 4-11 MHz multifrequency linear probes and $4-11 \mathrm{MHz}$ convex probes and recorded the findings on the hard discs of the devices. During the hospital stay all 154 patients underwent ultrasound with dynamic assessment at least three times, and in severe cases up to five times: on admission, on day 3-5 or day 5-7 of hospital stay depending on the severity of the case and treatment efficacy, then on completion of etiotropic therapy on day 10-14. After discharge, examination was repeated out-patiently at least two times per month, in separate cases the children were followed up for three months with a monthly twofold examination.

Children over 1 year of age underwent transthoracic ultrasound in plantigrade and recumbent positions, children under 1 year of age were examined only in decubitus. Anterior parts of the lungs and pleura were studied in a sitting position, with the child's shoulders fully straightened; lateral parts were examined with the
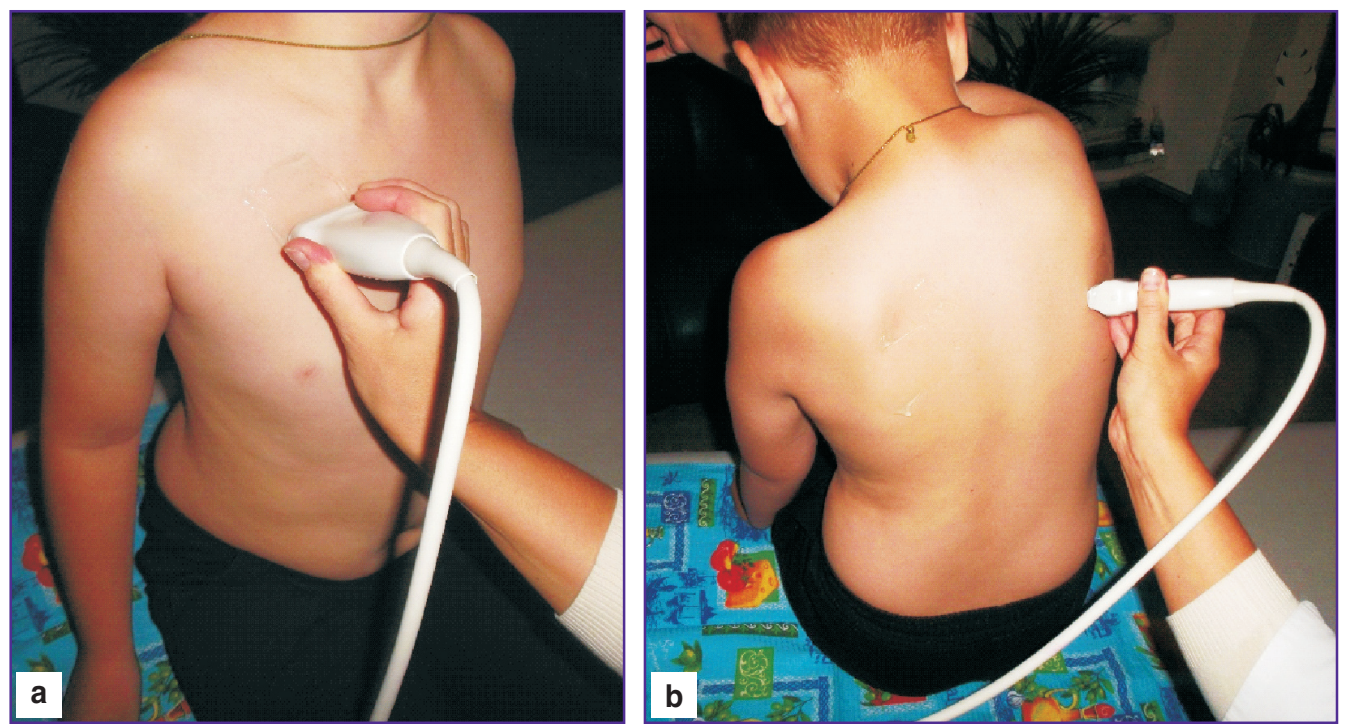

Figure 1. Lung ultrasound technique, transversal scanning of the lower (a) and upper (b) lobes from the intercostals space on the right 
child's arm up; posterior ones were examined with a slightly stooped back, and the examination of posterior parts of the upper lobes was performed with an additional alternate abduction of the arm obliquely upwards to the opposite side (Figure 1). Scanning was performed in the longitudinal and transversal planes moving the probe across the intercostal space with a smooth jiggling.

Examination was started with the upper posterior intercostal space, consecutively moving the probe from paravertebral parts to the lateral ones and further to the parasternal fields. Both halves of the chest were examined top-down in turns, paying special attention to the segments most frequently affected in each age group. When necessary, forced ventilation test was performed in children over 5 years of age to study respiratory mobility of the lungs. It was done by placing the probe above the lower edge of the lung perpendicular to the body surface, and determining the amplitude of its shifting in deep breathing.

While visualizing apneumatic (air-free) pulmonary tissue areas, we measured them in two mutually perpendicular planes, determined their localization in the lobes and segments, evaluated the contours, echogenicity and echostructure. In the echostructure we considered the quantity, the distribution pattern, and the form of hyperechogenic signals from the air, anechoic tubular structures and fluid inclusions. In case of pleural effusion its volume and echostructure, as well as the pleura state were assessed.

Diagnostic decision was made on the basis of clinical classification of pneumonia with the definition of the form and localization of the inflammatory process: focal, segmental or lobar pneumonia, indicating the damaged segment or lobe number, as well as the pleural cavity condition.

Results and Discussion. On objective examination the diagnosis of "pneumonia" was made to all 154 children. Among them 126 children (81.8\%) showed typical X-ray picture of the disease, and in the rest 28 children (18.2\%) pneumonias appeared $X$-ray-negative, and the diagnosis was confirmed by the findings of chest CT. Lung ultrasound performed regardless of the chest X-ray results revealed zones with pulmonary tissue airness impairment of various sizes. In 147 children (95.5\%) sonographic picture was complemented by clinical history-taking data of the inflammatory process which allowed us to diagnose pneumonia at first sonography. Only 7 children (4.5\%) showed disagreement of echosymptoms with the clinical picture, and required CT differential diagnosing.

The first chest ultrasound was performed on the day of admission to the hospital. The purpose of the investigation was to confirm clinical data by detecting the area of pulmonary tissue airness impairment and identifying it as the area of inflammatory infiltration, to determine the localization and the size of pneumonic focus, to assess the state of pleural cavity. The main indication to ultrasound was the information collected by history-taking, and absence of X-ray symptoms did not exclude scanning.

Morphologically, parenchymatous pneumonia is defined as lobar, polysegmental, segmental, bronchopneumonia (focal pneumonia) and pneumonia with confluent foci. According to echosemiotics we combined them in two groups differing in homogeneity and the size of apneumatic area and called them extensive and focal ultrasound type of inflammatory infiltration.

The extensive ultrasound type was revealed in 64 children (41.5\%) and included segmental (31; $20.1 \%$ ), polysegmental (23; $14.9 \%)$ and lobar (10; 6.5\%) pneumonias which had similar sonographic picture that differed only in the size and shape of the apneumatic area. The lesion extent was identified by the infiltrate projection on the thoracic wall according to the accepted lung division into lobes and segments. A specific feature of segmental pneumonias was a delta-shaped airfree area with the apex toward the root of the lung and the base toward the thoracic wall. Lobar pneumonias preserved the anatomic shape of the infiltrated parts of the lung.

In extensive pneumonia the inflammatory infiltration was located as a hypoechogenic area with heterogeneous structure due to linear hyperechogenic echoes representing reflection of the ultrasound from the air in the bronchi - an air echobronchogram (Figure 2). We detected it in 78 children (50.6\%), most often in a form of short segments radiating from the root to the periphery, rarely they were found at rather a great distance and were branching. The quantity and distribution of the echobronchogram elements depended on pneumonia severity and served as an important criterion of its

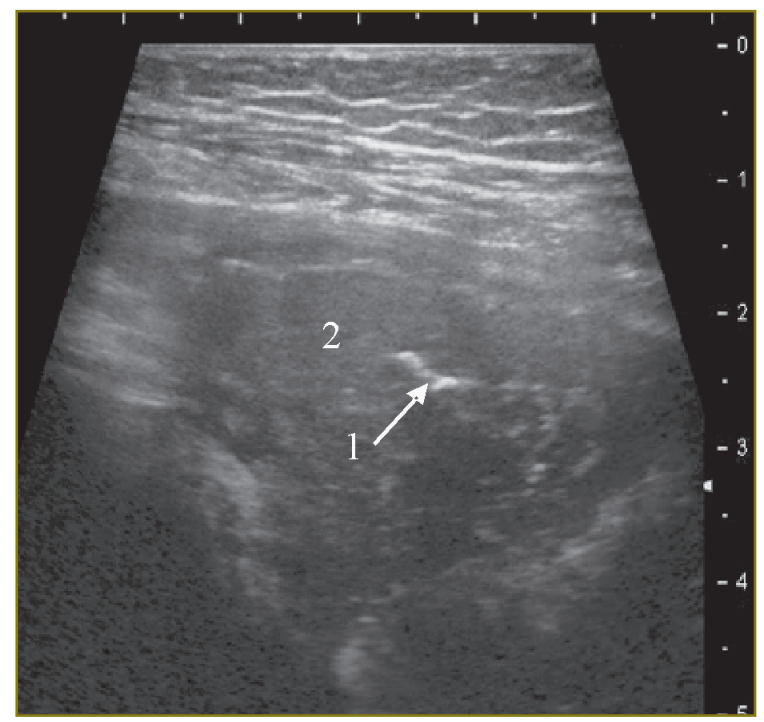

Figure 2. Segmental pneumonia echogram with elements of air echobronchogram (1) in hypoechogenic inflammatory infiltrate (2) 


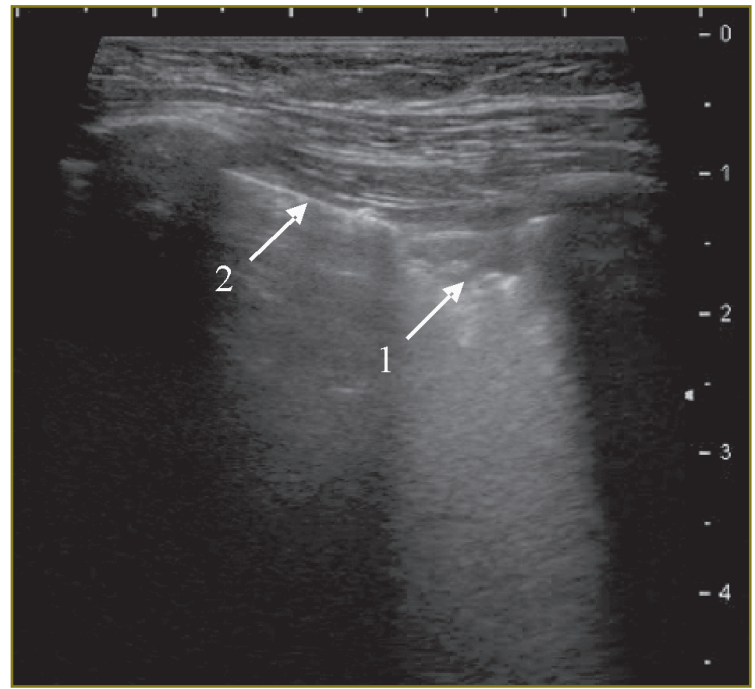

Figure 3. Echogram of focal pneumonia presented in the form of a small homogeneous hypoechogenic focus (1) with an irregular contour, surrounded by the air lung tissue (2)

regression. A symptomatic echobronchogram was a favorable prognostic sign of a rapid infiltrate resolution.

Pneumonia in children, especially those of younger age, almost always ran with different amounts of pleural effusion. Minimal volume of fluid was detected at the level of inflammatory infiltrate in the form of $2 \mathrm{~mm}$ thick anechoic slit-like space accompanied by the divergence of the pleural layers. In more severe cases 5 to $50 \mathrm{ml}$ of anechoic fluid was located in costodiaphragmatic recess and other parts of the pleural cavity.

Focal ultrasound type was revealed in 90 children $(58.4 \%)$ and included focal $(39 ; 25.3 \%)$ and focal confluent $(51 ; 33.1 \%)$ forms of pneumonias. It is characterized by small rounded and protracted subpleural foci $10-15 \mathrm{~mm}$ in diameter, with hypoechogenic structure and welldefined smooth contours (Figure 3).

Blood flow was not registered during color Doppler mapping. In focal pathomorphological form of pneumonia a single balloon-shaped pneumonic focus was located. In focal confluent pneumonia it presented an area with irregular, less clear contours extended along the lung surface, or several foci adjoining or closely situated but divided by the air lung tissue.

The terms of performing the second chest ultrasound were determined by a child's state in the course of treatment. In positive clinical dynamics it was performed on day $5-7$, in the negative one - already on day 3-5, and in case of a sharp worsening of the patient's condition - on the same day. The purpose of that study was to assess etiotropic therapy efficacy by the dynamics of the size and echostructure changes of the apneumatic area.

Dynamics was considered positive in case of infiltrate size reduction with airness restoration from the center to the periphery and in case of increase in the extension and the number of air echobroncogram elements. In focal ultrasound pneumonia the dynamics was positive in case of the decrease in focus contour clearness and greater intensity of the "comet-tail" artefacts at the boundary of the surrounding air lung tissue.

The signs of negative dynamics were increasing size of pneumonic infiltrate or pleural effusion volume, appearance of regions of destruction in the existing infiltrate or detection of new foci of pneumonia revealed in 7 patients $(4.5 \%)$. The absence of ultrasound signs indicating positive dynamics in 31 children $(20.2 \%)$ was considered a negative criterion as well.

If positive dynamics was noticed during the second examination, we performed the third chest ultrasound following 5 days, i.e. on day 10-14 of the disease, which often corresponded to the last day of etiotropic therapy. In case of good clinical dynamics and fast recovery the third ultrasound was performed at the physician's discretion shortly before or just on the day of the patient's discharge. In case of negative sonographic signs or lack of ultrasound dynamics at the second ultrasound etiotropic therapy had to be changed which was done in 38 children $(24.7 \%)$. So the third ultrasound examination was performed $5-7$ days after antibiotic replacement. Its purpose was to assess dynamic changes in the affected area size and sonographic picture after etiotropic therapy alteration.

If the child's condition was satisfactory, the forth chest ultrasound was performed in the outpatient setting not earlier than 2 weeks after hospital discharge (on day 20-25 of the disease), but if aggravation was noted in the course of recovery, ultrasound was done on the day of consulting a doctor. When hospital treatment was to be continued, the forth chest ultrasound was performed on the last day of the repeated etiotropic therapy.

Children with persistent focus were administered the fifth chest ultrasound after 2 weeks, and in complete airness recovery - after 1 month. Then a check-up ultrasound was performed in the outpatient setting after 3 months, if there was no apneumatic area in the previous sonography, but if it persisted, scanning was repeated monthly until complete recovery of the lung airness. After the fifth ultrasound apneumatic area was revealed only in 7 children (4.5\%) but in 13 patients $(8.4 \%)$ multiple "comet-tail" artefacts were located in the pneumonia area (Figure 4). Chest ultrasound was performed once in 3 months if the patient's condition was satisfactory, there was no apneumatic area, and "comettail" artefacts or pleural thickening remained.

"Comet-tail" artefacts are echo signs of interstitial lung changes of different etiology, including inflammatory ones. In interstitial pneumonia acute inflammation develops in the alveolar walls and interstitial tissue without primary exudation to the respiratory parts. Alveoli airness remains and inflammatory infiltrate does not form, therefore, ultrasound does not penetrate deeply 


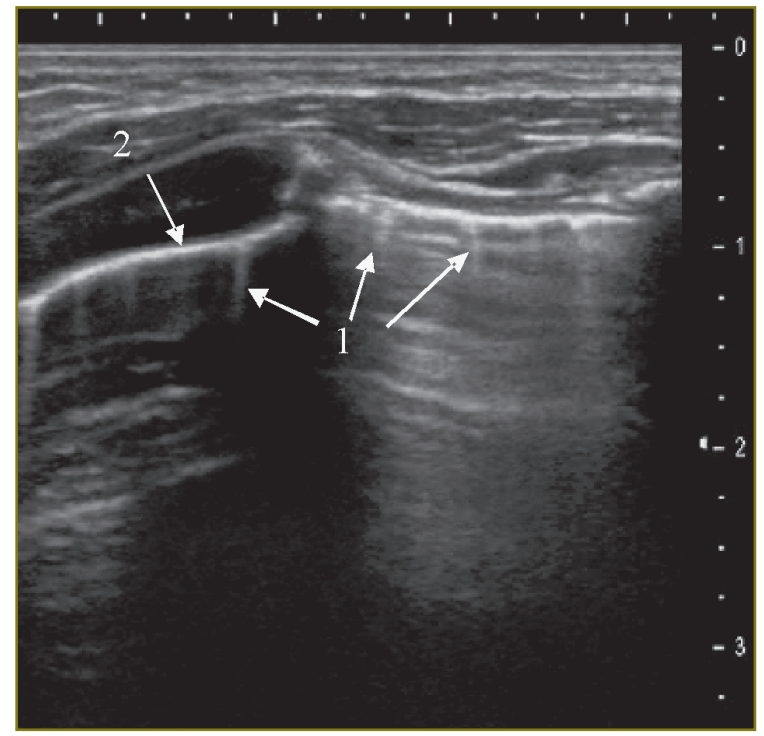

Figure 4. Interstitial pneumonia echogram: multiple "comettail" artefacts (1) behind hyperechogenic parietal line of the air lung (2)

to the lung which preserved its airness. Consequently, hyperechogenic parietal line does not disappear from its surface, it only alters, being the main ultrasound criterion of this form of pneumonia.

The lung surface in the area of interstitial inflammation looks like a wider and less clear hyperechogenic parietal line without typical "repeated echo" artefacts, but with multiple "comet-tail" artefacts (See Figure 3). As interstitium edema decreases, their intensity goes down and they disappear. Hyperechogenic parietal line becomes smoother, and when final recovery of the normal lung structure takes place, "repeated echo" artefacts appear again.

However they can occur not only in pneumonia but also in fibrous changes of intersticium in pneumosclerosis, bronchopulmonary dysplasia or pulmonary congestion due to heart failure. Interstitial pneumonia can be established only when there is a relevant clinical and laboratory picture, or in the course of dynamic observation of parenchymatous pneumonia, otherwise it is necessary to look for another cause of "comettail" appearance. Therefore, we consider the persisting echopicture of interstitial lung changes without dynamics during 6 months to be the indication for chest CT in order to exclude focal pneumofibrosis or bronchiectasis.

Analyzing age characteristics of pneumonia ultrasound image, it was found that in children of group 1 (from birthday to 3 months of age) focal processes in the form of hypoechogenic homogeneous foci up to $10 \mathrm{~mm}$ in size located in the posterior segments of the upper and lower lobes (segments 2, 6, 10) were detected most commonly. Larger inflammatory infiltrates within a segment were not so often revealed; lobar infiltration in this age group was not found. Air echobronchogram elements were visualized only when the infiltrate size was almost equal to that of a segment. It is necessary to note that even small inflammatory foci were often accompanied by the clinical picture of severe pneumonia.

Due to adequate and timely etiotropic therapy recovery of parenchyma airness developed faster as compared to the similar pneumonia types in children of other age groups. Nevertheless, interstitial changes in the form of hyperechogenic parietal line with "comet-tail" artefacts remained at the place of the resolved infiltrate for a longer time, at least a week more. Pleural effusion at the lesion side, both at the level of infiltrate and in pleural recess, was minimal, more often in the form of anechoic space up to $5 \mathrm{~mm}$ thick.

In children of group 2 (3 months to 3 years of age) anterior and posterior lung segments were affected with the same frequency, and mainly in the form of focal infiltrates of a bigger size, up to $20 \mathrm{~mm}$. Similarly, in this age group pneumonia was accompanied by a small volume of pleural effusion located not only at the infiltrate level but in the pleural recess as well. Clinical severity of the disease depended on the inflammatory infiltrate size to a more extent, and increased with the apneumatic area growth.

In children of group 3 (4 to 7 years of age) inflammatory infiltrates were commonly revealed in the middle lobe on the right-hand side and in the lingular segments on the left-hand side as well as in segments 10 on both sides, which is connected with the anatomic structure of the bronchial tree. These segments are worse ventilated by the bronchi, and pathogenic bacteria adhere and multiply there faster. Segmental forms of pneumonias with the rapid increase of inflammatory infiltration volume prevailed, when not treated in time. Probably, it is caused by intensive development of the Pores of Kohn at this age and increasing functional role of collateral ventilation contributing to the spread of infectious agent within a lobe.

Lobar pneumonia, in the structure of which air echobronchogram elements were always traced, occurred more frequently in this age group. Their lager quantity in the first days of etiotropic therapy was a favorable prognostic sign. Pleural effusion was always observed on the side of the lesion, and its amount grew dependent on the process duration.

In children of group 4 ( 7 to 18 years of age) lobar pneumonia with localization in the lower lobes and in the middle lobe on the right side prevailed with typical adult echosemiotics represented by air echobronchogram of different intensity at the hypoechogenic background of apneumatic lung tissue.

In groups 2 and 4 interstitial inflammatory changes were often seen in the lungs. If they were detected on both sides with irregular distribution over the lung surface in the form of separate areas and had a clinical picture of atypical pneumonia, interstitial pneumonia was diagnosed. It was always accompanied by a small 
amount of effusion in the pleural cavity. In 62 children $(40.2 \%)$ interstitial lung changes, revealed by transthoracic ultrasound examination were not confirmed by X-ray findings, but they disappeared in the course of etiotropic therapy and patient recovery.

Conclusion. Chest ultrasound is an informative and radiologically safe method of pneumonia diagnosis and its dynamic control in children of different age, which enables to assess timely the efficacy of etiotropic therapy. In the pneumonia echosemiotics focal and extensive ultrasound forms can be distinguished. Focal pneumonia is described as having a small rounded subpleural infiltrate with homogeneous structure, it is typical of newborns and younger children. An extensive type occupies a segment or a lobe and appears as delta-shaped apneumatic area with irregular contours and heterogeneous structure with an air echobronchogram, it occurring in children of older age. Assessment criteria of pneumonia regression are the size and echostructure of apneumatic area. In the course of hospital stay ultrasound examination should be performed at least three times: the first ultrasound is done on admission to establish pneumonia, and to determine the inflammatory infiltrate dimension and localization; the second ultrasound - on day $3-5$, or day $5-7$ to assess the dynamics of sonographic picture and the necessity of etiotropic therapy change if there is no positive dynamics; the third ultrasound is made before the discharge to evaluate residual effects and determine the follow-up strategy. Outpatient observation is necessary to control complete recovery of lung tissue airness and regression of interstitial changes in it.

Study Finding and Conflict of Interest. This research was not financed by any sources, and there are no conflicts of interest associated with the research.

\section{References}

1. Geppe N.A., Malakhov A.B., Volkov I.K., Kozlova L.V., Dronov I.A. On the problem of further development of research and practice program on community-acquired pneumonia in children. Russkiy meditsinskiy zhurnal - Pediatriya 2014; 22(3): 188-184.

2. Samsygina G.A. Infektsii respiratornogo trakta u detey rannego vozrasta [Respiratory infections in young children]. Moscow: Pul's M; 2013; 260 p.

3. Safonov D.V., Shakhov B.E. Ul'trazvukovaya diagnostika vospalitel'nykh zabolevaniy legkikh [Ultrasound diagnosis of pneumonia]. Moscow: Vidar-M; 2011; 120 p.

4. Reißig A., Görg C., Mathis G. Transthorakale sonografie bei der diagnostik pulmonaler erkrankungen: ein systematischer zugang. Ultraschall in Med 2009; 30(5): 438458, http://dx.doi.org/10.1055/s-0028-1109703.

5. Lee G.E., Lorch S.A., Sheffler-Collins S., Kronman M.P., Shah S.S. National hospitalization trends for pediatric pneumonia and associated complications. Pediatrics 2010; 126(2): 204213, http://dx.doi.org/10.1542/peds.2009-3109.

6. Dvoryakovskiy I.V., Odinaeva N.D., Potekhina T.V. Pulmonary sonography in new born children. Ul'trazvukovaya $i$ funktsional'naya diagnostika 2010; 6: 41-49.

7. Copetti R., Cattarossi L. Ultrasound diagnosis of pneumonia in children. Radiol Med 2008; 113(2): 190-198, http://dx.doi.org/10.1007/s11547-008-0247-8. 